\title{
ANÁlisis CUALITATIVO del diseño de estrategias en la transición de operaciones para el ensamble de productos en las empresas de Ciudad Juárez
}

\author{
José Éder Leal Lara \\ Universidad Autónoma \\ de Ciudad Juárez
}

ReCIBIDO: 19 DE NOVIEMBRE DE 2014 ACEPTAdo: 13 DE MARZo DE 2015

\section{RESUMEN}

$\tau$ a transición de operaciones para el ensamble de productos requiere de configurar y ordenar las operaciones por secuencias y grado de aprobación en las etapas que componen el proceso. La primera fase se lleva a cabo generalmente en una negociación, de la cual forman parte los corporativos dueños de los productos y las empresas que proveerán el servicio de ensamble. El enfoque principal de la transición de operaciones es minimizar el impacto negativo derivado de las demoras en el lanzamiento y liberación de los procesos donde se elaborarán los nuevos productos, con el propósito de que la producción en masa se despliegue una vez puesta en marcha su fase de inicio. La falta de una metodología para el diseño de estrategias en la gestión del proceso de transición de operaciones en las empresas de manufactura en Ciudad Juárez, se traduce en un bajo desempeño cuando se busca insertar los nuevos productos a las operaciones, generando también bajos niveles de competitividad en este sector.

Palabras clave: transición de operaciones, ensamble de nuevos productos, proceso, diseño de estrategias.

\section{Abstract}

$\mathrm{T}$

The operation transition for the products assembly required to configure and sort the operation activities by sequence and approval level in every single phase of the process. The first phase is usually performed in a negotiation by the corporate owners of the products and the companies that provide the assembly service. The main interest in the operation transition is to minimize the negative impact generated by delays in the launch and release pro- 
cesses where new products will be developed with the purpose of deploy the mass production in an effective way. The lack of methodology on design strategies in the operation transition management at the manufacturing companies in Ciudad Juárez brings as a result low operational performance when companies try to insert the new products at the operations, generating as well low competitiveness levels in this industrial sector.

Keywords: operation transition, new products assembly, process, strategy design.

\section{INTRODUCCIÓN}

$\mathrm{L}$ as empresas de manufactura en la actualidad operan bajo un entorno que resulta complejo para el manejo de los negocios. Por esta razón, la administración de las operaciones es un elemento fundamental para garantizar la efectividad de estos en la fase de inicio en la introducción de los nuevos productos. Las operaciones de las organizaciones se componen de una gama muy amplia de variables, que están involucradas directamente en el desempeño de las compañías; algunas de estas variables son la administración de la cadena de suministros, la administración del capital humano y otras series de actividades que deben ser administradas simultáneamente.

Un elemento esencial para lograr la permanencia de las empresas en los mercados, es la administración de la introducción de los nuevos productos que tendrán que ser manufacturados dentro de sus instalaciones. Por esta razón, la transición de operaciones para el ensamble de productos es fundamental para alcanzar la permanencia deseada por las compañías del sector. Para lograr este objetivo, es necesario que las empresas de manufactura diseñen estrategias eficientes para la transición de operaciones y la introducción de nuevos productos. En el desarrollo de este documento, se muestra la necesidad e importancia de administrar estos tipos de procedimientos en las compañías de manufactura locales.

\section{Metodología DE LA INVESTI- GACIÓN PARA LA TRANSICIÓN DE OPERACIONES}

$\mathrm{E}$ ste análisis descriptivo-cualitativo demuestra que, en la actualidad, la complejidad de los mercados está forzando a las empresas de manufactura a extender las dimensiones de los servicios que ofrecen. Las implicaciones gerenciales de las compañías para transferir los productos, parten de la idea de los corporativos de tener una relación cercana con la cadena de valor de los clientes (Nogueira, Proença, Spencer, \& Cova, 2013).

Las empresas de manufactura de Ciudad Juárez deben ajustarse a la dinámica con respecto a la transición e introducción de nuevos productos, con el propósito de mejorar su competitividad y ampliar el panorama de posibles nuevas inversiones, ofreciendo a los clientes potenciales una rápida inserción de los productos en las operaciones por parte de las organizaciones proveedoras del servicio, de acuerdo a Heizer, \& Render (2009), quienes puntualizan:

[...] En algún momento, un producto, ya sea un bien o un servicio, fue seleccionado, diseñado y definido, progresó desde una idea hasta definición funcional y, después, quizá a un diseño. Ahora la administración debe tomar la decisión de si continuará con su desarrollo y producción o dar por terminada la idea del producto.
Una vez tomada la decisión, casi siempre hay un periodo de pruebas de producción para asegurar que sea factible producir el diseño, esta es la prueba de manufacturabilidad. Esta prueba también brinda al equipo de operaciones la oportunidad de desarrollar las herramientas necesarias, los procedimientos de control de calidad, y la capacitación del personal para asegurar que la producción se inicie de manera exitosa (p. 178).

La transición de operaciones en las empresas de manufactura está conformada por un proceso de tres etapas. La primera está enfocada a los objetivos del mercado; la segunda, a los intereses del producto, y la última etapa, al enfoque de los factores de valoración de la producción (Erhun, Gonçalves, \& Hopman, 2010). Para las compañías de manufactura en Ciudad Juárez, el proceso se compone también de tres etapas: la negociación entre corporativos, las corridas piloto y la producción en masa. Como se muestra en la figura 1 , cada una de las etapas contiene elementos específicos necesarios para lograr la secuencia de operaciones que el proceso de transición requiere.

Algunos elementos necesarios y primordiales del proceso, se enlistan a continuación:

- Etapa 1: capacidad instalada en la planta; especificaciones del producto y procesos; diseño de sistemas de calidad; establecimiento de estándares de producción; diseño de la distribución física; información general de materiales y equipo; y por último, la integración y desarrollo del personal requerido en los procesos de manufactura.

- Etapa 2: establecimiento de la curva de aprendizaje necesaria con el fin de robustecer el entrenamiento sobre el producto y los procesos; 
contar con el total de los materiales en la planta; construir cantidades pequeñas del producto; analizar todos los procesos diseñados y utilizados para la manufactura, con el objetivo de mejorarlos antes de iniciar con la producción en masa.

- Etapa 3: generar grandes cantidades del producto; mantener los niveles de inventario de la materia prima, así como la recertificación del personal con respecto a sus funciones.

Krajewski, Ritzman, \& Malhorta (2010) mencionan al respecto:

[...] El proceso de negociación se enfoca en obtener un contrato efectivo que involucra precio, calidad y requerimientos de entrega en el proceso interno del cliente y los proveedores, la naturaleza de las relaciones que se realiza con los proveedores puede afectar la calidad, tiempo de respuesta, el precio y el servicio de los productos (pp. 364-365).
Según Erhun et al. (2010), la transición de operaciones falla cuando no se conocen los componentes que conforman las etapas del proceso de transición de los nuevos productos, generando problemas financieros no contemplados durante el proceso de introducción.

Los impactos negativos en las finanzas con respecto a las operaciones, a consecuencia de una pobre gestión en las etapas del proceso de transición, generan barreras operativas en los siguientes elementos clave en el proceso de transición:

Estas barreras son generadas en las etapas de las corridas piloto y la producción en masa, las cuales se describen en la figura 1 .

El ciclo de vida de los productos es relativamente corto, lo cual origina que:

a) La transición de los productos sea un desafío para las empresas de manufactura;

b) El rango de vida de los productos puede estar entre 12 y 24 meses; y

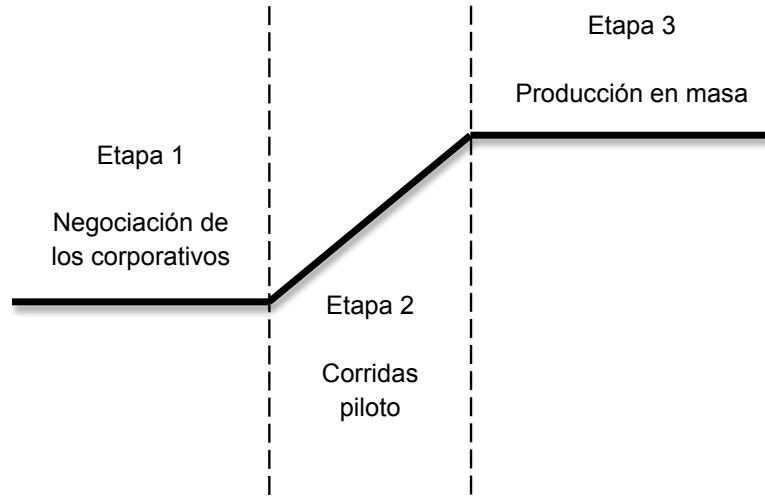

Figura 1. Etapas de la transición de operaciones o introducción de nuevos producto Fuente: elaboración propia con base en: Erhun et al. (2010). c) Financieramente pueden representar alrededor de un tercio de las ganancias anuales de las compañías de este sector ( $\mathrm{Li}$, Graves, \& Rosenfield, 2010).

Si la gestión de estos elementos resulta ser ineficiente, se generará pérdida de competitividad asociada con la transición de operaciones (Gopal, Goyal, Netessine, \& Reindorp, 2011); este tema ha sido poco estudiado por la literatura de la administración de operaciones y tampoco se ha cubierto en los libros de desarrollo de nuevos productos.

Por otra parte, de acuerdo a Langford (2007), los principales factores que afectan la pobre competitividad en la transición de operaciones son los costos operativos, así como el sitio para ubicar las plantas de manufactura. Según este autor, el proceso decisional que se contempla para seleccionar la mejor ubicación de la planta que manufacturará los nuevos productos, debe realizar un análisis y escrutinio a través de factores relacionados con la disponibilidad de materiales y los centros de mercado. Los elementos básicos para la selección son:

1) Costos de transportar la materia prima.

2) Costos de transportar los productos terminados de las empresas de manufactura a los centros de mercado.

3) Costos de producción (laborales, impuestos, utilidades y de mantenimiento).

Las empresas de manufactura juarenses poseen la mayor parte de los elementos mencionados por Langford (2007), que son requeridos para soportar las operaciones que las compañías extranjeras y nacionales requieren para elevar los niveles de competitividad con respecto a la manufacturación de los productos y la 
distribución, principalmente en Estados Unidos de un centro comercial principal, para las empresas que operan en Ciudad Juárez.

Las empresas de manufactura extranjeras y nacionales demandan a la zona fronteriza la creación y mantenimiento de infraestructura, para poder soportar la gestión de sus operaciones y mejorar con ello sus costos y eficiencia operativos. La infraestructura, sin duda, es un componente ajeno a las operaciones internas de las compañías de manufactura, pero indispensable para poder incrementar su competitividad a nivel global.

Entre los países latinoamericanos, México tuvo el peor desempeño ante la crisis por sus políticas públicas inadecuadas, principalmente por el aumento a los impuestos en la producción y el consumo, que generaron el deterioro económico de sus habitantes e impactó negativamente el ámbito del trabajo (Castilla Ramos, \& Torres Góngora, 2010); la transición de operaciones y, por consecuencia, la introducción de los nuevos productos, se vieron sumamente afectadas, lo que ocasionó que México perdiera competitividad ante el resto de los países que ofrecen el servicio de manufactura.

Las empresas de manufactura en Ciudad Juárez, para enfrentar la competencia doméstica e internacional, requieren de insertar en sus operaciones productos extranjeros y nacionales, así como materia prima o componentes para mejorar su ventaja competitiva (Padilla-Pérez, \& Martínez-Piva, 2009); si la cantidad de productos o de familias de productos que se transfieren en las compañías de manufactura, es constante durante los años, refleja cierto grado de competitividad en las mismas, debido a que se puede apreciar un grado de confiabilidad deseable de los clientes con respecto a la manufacturación de los productos.
La transición de operaciones, sin duda, tiene elementos asociados a la manufactura y distribución de los productos, que incrementan los costos de producción; algunos de estos componentes son laborales, impositivos, de utilidades y de mantenimiento.

La proximidad de los sitios de producción a los centros de mercado se traduce en bajos costos de transportación de los productos terminados, por otro lado la falta de cercanía a los centros de mercado incrementa los costos de transportar los productos terminados (Langford, 2007, p. 329)

La ventaja de la cercanía de las empresas de manufactura que operan en Ciudad Juárez con respecto a uno de los principales mercados del mundo, Estados Unidos, son los bajos costos de transportación que se requieren en la distribución y logística, en general, de los productos terminados, lo cual contribuye a que las compañías de manufactura logren incrementar su productividad y rentabilidad gracias a la gestión efectiva de las operaciones.

\section{DESARROLLO DE LA INVESTIGA- CIÓN EN EL DI- SEÑO DE ESTRA- TEGIAS PARA LA TRANSICIÓN DE OPERACIONES}

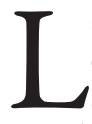
as empresas de manufactura requieren de procesos que puedan ser utilizados en la gestión y diseño de estrategias para la transición de operaciones, lo que resulta ser una actividad compleja, debido a las decisiones que los administradores deben tomar con respecto a qué tipo de estrategia diseñar y utilizar, contemplando para esta los cambios que sufren los productos y los procesos generados por el ambiente global y competitivo en el que se ofertan.

La importancia de los modelos estratégicos en la transición de operaciones, es que son indispensables para estructurar y darle prioridad a la secuencia de las actividades de los procesos operativos, que ayuden a diagnosticar el rendimiento de las empresas, para que se pueda tener una perspectiva del comportamiento de las operaciones una vez que la estrategia ha sido implementada. La estructuración descentralizada necesaria para cualquier actividad, se ha vuelto primordial para poder lograr los objetivos generales y específicos en las estrategias de las organizaciones (Drucker, 2003).

La escuela de posicionamiento de Porter (1997), ha sido utilizada por las empresas para estructurar sus estrategias y cimentar las actividades operativas, con el fin de lograr el posicionamiento y la permanencia en los mercados, para intentar alcanzar los objetivos de rentabilidad planteados en la fase de inicio de la estrategia. Porter (1997) menciona que “...esencialmente, la definición de una estrategia competitiva consiste en desarrollar una amplia fórmula de cómo la empresa va a competir, cuáles deben de ser sus objetivos y qué políticas serán necesarias para alcanzar tales objetivos" (p. 16), por lo que la integración de objetivos y políticas más los recursos con los que cuentan las compañías, son la fase inicial para trabajar con la formulación de estrategias exitosas que impacten positivamente el rendimiento operacional de las organizaciones, a través de la transición de operaciones.

De acuerdo a lo anterior, Corbett (2008) menciona que las estrategias para la manufactura de los productos tienen que adaptarse y ser configuradas contem- 
plando componentes internos y externos: en lo interno, el involucramiento de todas las áreas funcionales de la empresa y en lo externo, clientes y proveedores, para ligar exitosamente todos los elementos que la estrategia requiere. Pero la inconsistencia principal de los elementos internos que integran las estrategias en la mayoría de las compañías locales, es que carecen de una estructura sólida cuando surgen los primeros bosquejos estratégicos, propiciando que se postergue el tiempo de implementación de la misma e impactando negativamente en las operaciones internas de las organizaciones y en la introducción de nuevos productos.

Las estrategias en las empresas de manufactura en relación con la transición de operaciones son diseñadas para determinar cómo serán utilizados los bienes con los que cuentan para la introducción de nuevos productos; de igual manera, para estructurar las actividades por nivel de importancia operacional y financiera buscando obtener un desempeño óptimo y facilitar el logro de los objetivos en las organizaciones (Miltenburg, 2009).

Las estrategias en el proceso de la transición de operaciones, se inician con la formulación o establecimiento de actividades y negociaciones entre corporativos, y terminan cuando el producto es liberado a los departamentos de Manufactura y Producción, lo cual ocurre después de realizar una exhaustiva revisión de todas las actividades y elementos, tanto internos como externos, de los procesos, que fueron contemplados en el diseño de las estrategias durante la transición de operaciones.

Comprender la importancia de los objetivos estratégicos y la correlación de las actividades operacionales para la inserción de nuevos productos ayuda a determinar teóricamente los modelos estratégicos de manufactura implemen- tados por las empresas, como describe Miltenburg (2009), citando a Wheelwright, Vickery, Kim, Arnold y Platts. En la literatura de manufactura existen diferentes modelos estratégicos que las organizaciones deben analizar para estructurar sus estrategias, como se muestra en la tabla 1.

El proceso para el diseño de estrategias de manufactura deberá ser estandarizado y capaz de adaptarse a las necesidades de los clientes y de las empresas. El principio de flexibilidad en el diseño de estrategias, es un componente esencial en este proceso (Dimitrova, \& Rosenbloom, 2010). El grado de flexibilidad que posea cada estrategia dependerá específicamente de las necesidades operativas de cada una de las áreas funcionales involucradas en los proyectos de esta naturaleza; también es necesario romper las barreras operativas como el comportamiento organizacional, rigidez operativa institucional, así como la fragmentación funcional, como establecen Schroeder, Shah, \& Xiaosong Peng (2011).

Las empresas de manufactura seleccionan diferentes métodos para implementar y desarrollar sus estrategias, proponiendo, a su vez, mediciones y niveles de competitividad. Las medicio- nes y los niveles de competitividad son fundamentales para arraigar estrategias sólidas y efectivamente estructuradas, con el objetivo de soportar las condiciones del ambiente operativo interno y externo de las compañías de manufactura. "El desempeño de las empresas depende de cada una de las funciones que deben estar dirigidas al objetivo principal de la organización" (Drucker, 2001, p. 112). Por esta razón, las estrategias diseñadas para la transición de operaciones requieren forzosamente ser estructuradas desde los lineamientos y metodologías operacionales diseñados específicamente para las empresas de manufactura.

\section{Conclusiones}

$\mathrm{E}$ 1 diseño de estrategias en la transición de operaciones, es un proceso clave para las finanzas en las empresas de manufactura. Los procesos utilizados para la introducción de nuevos productos requieren de estrategias efectivas, que se enfoquen en reducir el impacto negativo en el desempeño y despliegue de los procesos que serán utilizados para ensamblar los productos; la efectividad de estos procesos contribuye

\section{Tabla 1. Modelos estratégicos en la literatura de manufactura}

\begin{tabular}{|l|l|}
\hline W1 & Estrategias de negocios \\
\hline W2 & Prioridades competitivas de manufactura \\
\hline W3 & Estructura e infraestructura de sistemas de manufactura \\
\hline K3 & Objetivos de manufactura \\
\hline K4 & Planes de acción \\
\hline H2 & Requerimientos del mercado \\
\hline H4 & Capacidad de manufactura \\
\hline H5 & Lo mismo que W3 cuando los objetivos son alcanzados \\
\hline
\end{tabular}

Fuente: Miltenburg (2009). 
a generar competitividad en este tipo de organizaciones.

Las negociaciones corporativas son la fase inicial del proceso de transición de operaciones, en las cuales se establecen los términos, especificaciones y costos para el ensamble de los productos. Cuando esta etapa finaliza, las empresas que proporcionarán el servicio de ensamble iniciarán con el diseño formal de los procesos de manufactura; esta etapa es conocida como el periodo de prueba o corridas piloto y su propósito es depurar los procesos diseñados, así como los entrenamientos que el personal requiere para ejecutar el ensamble de los productos y con ello garantizar la productividad en la última fase del proceso de transición de operaciones, siendo esta la liberación de los procedimientos que serán utilizados para ejecutar la producción en masa. La fase de la producción en masa en las empresas de manufactura, es la que genera el sustento financiero para los corporativos $y$, por ende, la generación de competitividad en este tipo de compañías.

Se ha logrado identificar con base en experiencia propia que algunas de las empresas de manufactura en Ciudad Juárez, no cuentan con una estructura institucional que les permita diseñar estrategias e implementar los procesos de transición de operaciones, además de la introducción de nuevos productos, desencadenando un pobre desempeño operacional, así como la pérdida de competitividad, impactando negativamente las finanzas de los corporativos.

\section{BibliografíA}

Castilla Ramos, B., \& Torres Góngora, B. (2010). Crisis sobre crisis en la in- dustria maquiladora de exportación y sus consecuencias en la ocupación. El Cotidiano, 162, 43-56.

Corbett, L. (2008). Manufacturing Strategy, the Business Environment, and Operations Performance in Small Low-tech Firms. International Journal of Production Research, 46(2), 5491-5513.

Dimitrova, B., \& Rosenbloom, B. (2010). Standardization versus Adaptation in Global Markets: is Channel Strategy Different? Routledge, 157-176.

Drucker, P. (2001). The Essential Drucker. Nueva York: Ed. HarperCollins Publishers.

----- (2003). El management del futuro. Buenos Aires: Ed. Sudamericana.

Erhun, F., Gonçalves, P., \& Hopman, J. (2010). Moving from Risks to Opportunities: a Process to Manage New Product Transitions. Stanford University, 1-20.

Gopal, A., Goyal, M., Netessine, S., \& Reindorp, M. (2011). The Impact of New Product Introduction Plant Productivity in the North America Automotive Industry. INSEAD, $1-34$.

Heizer, J., \& Render, B. (2009). Administración de las operaciones. México: Ed. Pearson.

Krajewski, L., Ritzman, L., \& Malhorta, M. (2010). Operations Management. New Jersey: Ed. Pearson.

Langford, J. (2007). Logistics Principals and Applications. Nueva York: Ed. McGraw-Hill.

Li, H., Graves, S., \& Rosenfield, D. (2010). Optimal Planning Quantities for Product Transition. Production and Operations Management, 19(2), 142-155.

Miltenburg, J. (2009). Setting Manufacturing Strategy for a Company's International ManufacturingNetwork.
International Journal of Production Research, 47(22), 6179-6203.

Nieto, A. (2013). Desafíos de la competitividad en la frontera norte de México en el marco del reajuste global. Frontera Norte, 25(50), 213221.

Nogueira, F., Proença, J., Spencer, R., \& Cova, B. (2013). The Transition from Products to Solutions: External Business Model Fit and Dynamics. Elsevier, 1093-1101.

Padilla-Pérez, R., \& Martínez-Piva, J. (2009). Export Growth, Foreign Direct Investment and Technological Capability Building under the Maquila Model: Winding Roads, Few Intersections. Science and $\mathrm{Pu}-$ blic Policy, 36(4), 301-315.

Porter, M. (1997). Estrategia competitiva. México: Ed. Continental, S. A. de C. V.

Schroeder, R., Shah, R., \& Xiaosong Peng, D. (2011). The Cumulative Capability "Sand Cone" Model Revisited: a New Perspective for Manufacturing Strategy. International Journal of Production Research, 49(16), 4879-4901. 